\title{
MICROCIRCULATION IN THE AGGREGATED LYMPHOID NODULES IN PIG
}

\author{
Katerina Kikalova, Jiri Charamza, Miroslav Kutal
}

\begin{abstract}
Department of Normal Anatomy, Faculty of Medicine and Dentistry, Palacký University Olomouc, Hněvotínská 3, 77515 Olomouc, Czech Republic; e-mail: kikina@tunw.upol.cz
\end{abstract}

Received: September 19, 2006; Accepted: November 12, 2006

Key words: Peyer's patches/Pig/Microcirculation

Aims: To compare the blood supply of the Peyer's patches in pigs with the already defined rodent one.

Materials and methods: Pig tissue was used. Injections of india ink, microscopic examination of the histological preparations stained by a haematoxylin - eosin and scanning electron microscopy of the corrosive casts were used for the depiction of the vessels.

Results: A model of the blood microcirculation of PP and its relation to the blood circulation of the small intestine was created. Only the capillaries in the follicles, but not the ascending arterioles as in rats were found.

\section{INTRODUCTION}

Aggregated lymphoid nodules - Peyer's Patches (PP) are secondary lymphoid organs in the small intestine, which are components of the gut - associated lymphoid tissue. They are typical for mammals and play an important role in the process of oral antigen stimulation. Pigs (Sus scrofa f. domestica) have, on average, 10-15 Peyer's Patches in their small intestine. ${ }^{1}$ The blood PP supply originates from the superior mesenteric artery via jejunal and ileal arteries and the ileocolic artery. The microcirculation in PP is important both for assuring intense metabolic demand, of the crypts and germ centres and for the circulation of the lymphatic cells. The blood microcirculation has been described at rats, guinea pigs and rabbits. ${ }^{2,3,5}$ The purpose of our study was to compare the blood supply of $\mathrm{PP}$ in pigs with already defined rodent ones.

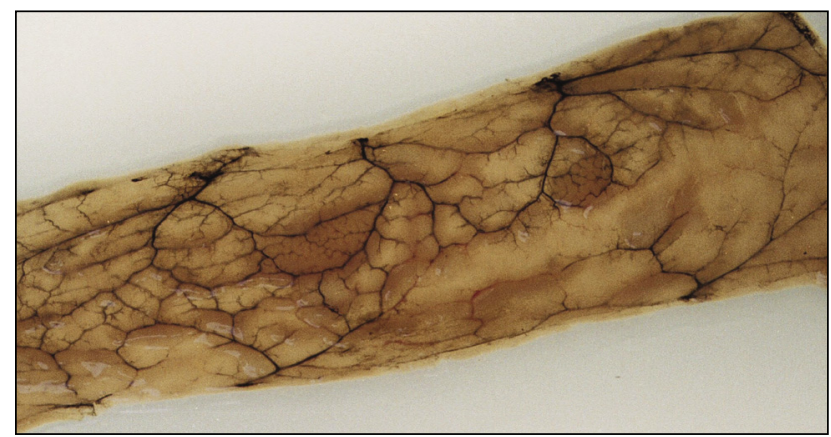

Fig. 1. Veins of the PP filling india ink. Serosal surface. Sample 5 x $10 \mathrm{~cm}$.

\section{MATERIALS AND METHODS}

The small intestine samples were taken from 6-weekold both male and female pigs which had been obtained from a slaughterhouse. Animals were kept 12 hours without food before they died. The parts of the small intestine including its arterial supply were cut out. Jejunal and ileal veins were flushed with india ink. Subsequently the intestine was photographed and cut into sections. Preparations obtained for histological treatment were $10 \times 6 \mathrm{~mm}$. They were stained by a haematoxylin-eosin and observed by a Carl Zeiss Jena microscope. Pictures were made by a Nikon Coolpix 4500 camera.

Furthermore we used a corrosion casts method. Veins were filled in the same way but resin (Dentakryl - Spofa) was used. After the polymerization preparations were immersed in a salt acid to get a corrosion of the organic

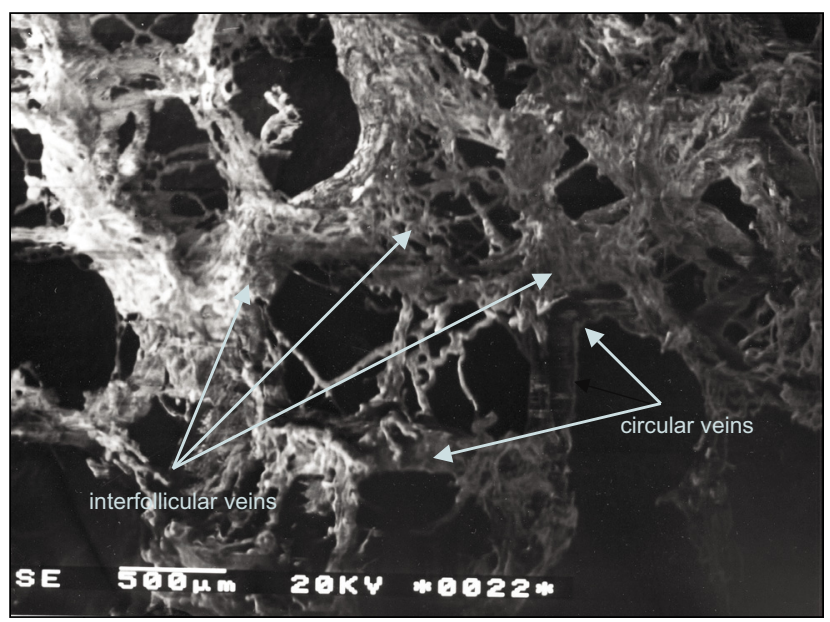

Fig. 2. SEM casts of the veins, magnification $46 \mathrm{x}$. 
tissue. Then they were dried, coated by a $10 \mathrm{~nm}$ layer of gold and palladium in a sputtering device Polaron E 5100 and examined under a Tesla BS 340 scanning electron microscope.

\section{RESULTS}

We observed macroscopically straight arteries and veins, which arrived together in the mesentery. These vessels divided into two after their achievement of the intestinal margin, likewise the peritoneum. Both arteries and veins branched and made an arterial and venous network within the intestinal wall. Vessels were clearly visible only on the serosal surface, whereas they were masked by the mucosa on the internal surface. Since Peyer's patches were located opposite the root of the mesentery, they were supplied from both sides. There was an irregular circuit around the periphery of PP, which was associated with several branches (Fig. 1). The veins, which had been filled by india ink, constituted little circles around each of the lymphatic follicles. During the process of scanning electron microscopy (SEM) we have observed, that these circular veins had common parts, which were in a contact (likewise as a honeycomb) (Fig. 2).

During the microscopical examination we observed the largest number both arteries and veins in submucosa. Arteries and arterioles were narrower and more linear than the relevant veins and venules. The slender vascular networks passed off the subserosa, lamina propria and between circular and longitudinal muscular layer. In follicles there were only capillaries. These pierced the follicles mostly horizontally and led into the postcapillar veins in the interfollicular area. SEM of the resin casts depicted numerous interfollicular veins, which led into the circular veins (Fig. 2).

We created the following proposed diagram of the integration of the blood microcirculation of PP into the blood supply of the small intestine (Schema 1).

\section{DISCUSSION}

The blood circulation of the lymphoid organs is appropriate to the arrangement of the lymphoid tissue in the pertinent organ. Thymus, lien and lymph nodes have an independent and very specific organization of blood supply, which reflects their functions. The microcirculation of the isolated and aggregated lymphoid nodules is an entire component of the intestinal blood circulation and it is liable to all effects, which can influence its.

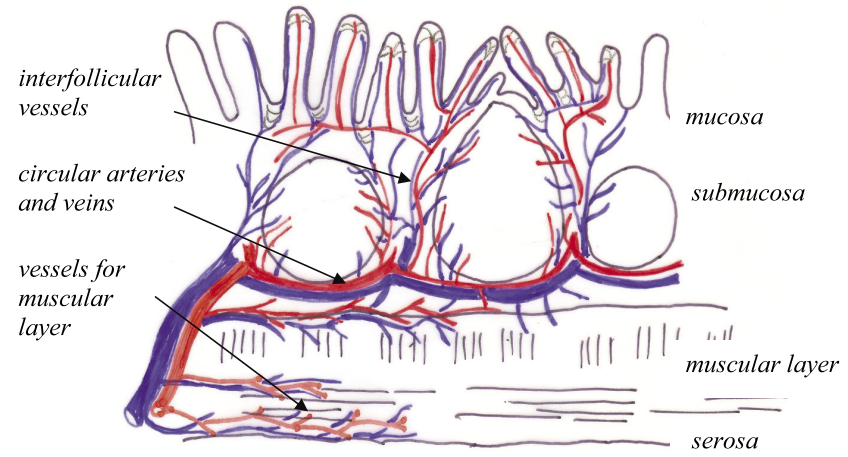

Schema 1. Model microcirculation in PP in pigs.

The postcapillary veins with the high endothelium are typical of the microcirculation in the lymphoid tissue. These veins are located in the interfollicular areas of PP.

Bhalla et al. described the PP microcirculation in rats. They described the ascending arterioles in the lymphatic follicles, which gave capillaries beneath the surface of the follicles, and subsequently led to the interfolicular veins. ${ }^{2}$ In contrast, Blau et al. observed only innumerable capillaries in the follicles of PP in guinea-pigs. ${ }^{3}$ As well as in guinea-pigs, follicles in the perirectal lymphoid tissue of rodents were supplied from the arterioles around the periphery in the follicles which gave the capillaries beneath the surface of the follicles. ${ }^{4}$ Yamaguchi and Schoefl described the microcirculation at PP in mice. The mouse nodules were permeated by a meshwork of the fine capillaries that were supplied by the arterioles entering on the serosal and lateral surface. ${ }^{5}$

We examined only the capillaries in the lymphatic follicles. However since we studied only the corrosion casts of the veins, our results are not complete.

\section{REFERENCES}

1. Pospischil A. Structur und funktion von Peyer'schen platten im darm verschiedener tierarten. Schweiz. Arch. Teirheilk. 1989; 131: 595-603.

2. Bhalla DK, Murakami T, Owen RL. Microcirculation of Intestinal Lymphoid Follicles in Rat Peyer's Patches Gastroenterology 1981; 81:481-491.

3. Blau JN. A comparative study of the microcirculation in the guineapig thymus, lymph nodes and Peyer's patches. Clin Exp Immunol. 1977; 27(2):340-347.

4. Kardon RH, Kessel RG. The Microcirculation of Lymphoid Tissue in Three Dimensions: Scanning Electron Microscopy of Corrosion Casts of the Lymph Node, Thymus, and Peri-rectal Lymphoid Tissue. Biomedical Research 2, Supplement, 1981; 173-179.

5. Yamaguchi K, Schoefl GI. Blood vessels of the Peyer's patch in the mouse: I. Topographic studies. The Anatomical Record 1983; 206(4):391-401. 